\title{
A numerical study of the correspondence between paths in a causal set and geodesics in the continuum
}

\author{
Raluca Ilie $^{1}$, Gregory B Thompson ${ }^{2}$ and David D Reid ${ }^{3}$ \\ Department of Physics and Astronomy, Eastern Michigan University, Ypsilanti, MI 48197, USA \\ E-mail: dreid@uchicago.edu
}

Received 13 December 2005, in final form 22 February 2006

Published 19 April 2006

Online at stacks.iop.org/CQG/23/3275

\begin{abstract}
This paper presents the results of a computational study related to the pathgeodesic correspondence in causal sets. For intervals in flat spacetimes, and in selected curved spacetimes, we present evidence that the longest maximal chains (the longest paths) in the corresponding causal set intervals statistically approach the geodesic for that interval in the appropriate continuum limit.
\end{abstract}

PACS numbers: 04.60.-m, 04.60.Nc

(Some figures in this article are in colour only in the electronic version)

\section{Introduction}

The causal set program proposes one of a number of approaches to the problem of quantum gravity [1-3]. A key aspect of this approach is that it postulates that spacetime is discrete rather than continuous. A causal set is a set $C$ of elements $x_{i} \in C$, and an order relation $\prec$, such that the set $C=\left\{x_{i}, \prec\right\}$ obeys properties which make it a good discrete counterpart for continuum spacetime. These properties are that (a) the set is transitive: $x_{i} \prec x_{j} \prec x_{k} \Rightarrow x_{i} \prec x_{k}$; (b) it is noncircular, $x_{i} \prec x_{j}$ and $x_{j} \prec x_{i} \Rightarrow x_{i}=x_{j}$; (c) it is locally finite in the sense that the number of elements between any two related elements $x_{i} \prec x_{j}$ is finite, i.e., $\left|\left[x_{i}, x_{j}\right]\right|<\infty$ and (d) it is reflexive, $x_{i} \prec x_{i} \forall x \in C$. The action of the order relation is to mimic the causal ordering of events in macroscopic spacetime. Since all events in spacetime are not causally related, not all pairs of elements in the set are ordered by the order relation. Hence, a causal set is a partially ordered set.

The continuing development of the causal set program can be roughly divided into two categories: kinematics and dynamics. Development of a causal set dynamics is the ultimate

1 Present address: Department of Atmospheric, Oceanic, and Space Sciences, University of Michigan, Ann Arbor, MI 48109, USA.

2 Present address: Department of Physics and Astronomy, University of Toledo, Toledo, OH 43606, USA.

3 Present address: Department of Physics, University of Chicago, 5720 S. Ellis Avenue, Chicago, IL 60637, USA. 
goal of this program and would represent its version of a theory of quantum gravity. However, the ability to investigate the implications of this dynamics will require development of the mathematical and computational tools needed to describe the physics of causal sets. This collection of techniques make up causal set kinematics. Of particular interest here is the correspondence between spacetime as a causal set and macroscopic spacetime. If a causal set comprises the true structure of spacetime it must produce a four-dimensional Lorentzian manifold in macroscopic limits.

The goal of determining how we can recognize and extract manifold-like properties from a partially ordered set is a kinematic issue of significant current interest. This paper reports on a numerical study concerning one aspect of this goal; specifically, we address the correspondence between paths in a causal set and geodesics in continuum spacetimes. To the best of our knowledge, this is the first published investigation of this correspondence that includes curved spacetimes. In what follows, we first describe the key ideas related to this correspondence, we then describe the details of how our calculations were performed and finally we present our results and conclusions.

\section{The path-geodesic correspondence}

The properties of causal sets stated previously do not guarantee that the sets will display the manifold-like behaviour required by the physical theory of causal sets we ultimately seek. In fact, it is well known that most sets satisfying those properties will not be consistent with points in a spacetime manifold. A necessary (but not sufficient) requirement for a causal set to be like a manifold is that it can be embedded into a manifold uniformly with respect to the metric. An embedding of a causal set is a mapping of the set onto points in a Lorentzian manifold in such a way that the lightcone structure of the manifold preserves the ordering of the set. The most probable embeddings will be uniform if the mapping corresponds to selecting points in the manifold via a Poisson process. When this latter requirement is met, the embedding is said to be faithful.

Recall that the length of the geodesic between two causally related events gives the longest proper time between those events. To see what the most natural analogue to geodesic length is for causal sets we must first define a few terms. A link, $\preceq$, in a causal set is an irreducible relation; so, $x_{i} \preceq x_{k}$ iff $\nexists x_{j} \ni x_{i} \prec x_{j} \prec x_{k}$. A chain in a causal set is a set of elements for which each pair is related; for example, $x_{a} \prec x_{b} \prec \cdots \prec x_{z-1} \prec x_{z}$ is a chain from $x_{a}$ to $x_{z}$. A maximal chain, or path, is a chain consisting only of links, such as $x_{a} \preceq x_{b} \preceq \cdots \preceq$ $x_{z-1} \preceq x_{z}$.

Myrheim argued [4] that the length of the longest path between two related elements in a causal set is the most natural analogue for the geodesic length between two causally connected events in spacetime. The length of a path is defined to be the number of links in that maximal chain. A more formal statement, that we refer to as the Myrheim length conjecture, is as follows:

Let $\left\{C_{n}\right\}$ be a sequence of causal sets $C_{n}=\left\{x_{i}, \prec\right\}$ of increasing density $\rho$ that are faithfully embeddable into a Lorentzian manifold $\mathcal{M}$ by a map $g: C_{n} \rightarrow \mathcal{M}$. Then, in the limit $\rho \rightarrow \infty$, the expected length of the longest maximal chains between ordered pairs $\left(x_{i}, x_{j}\right) \in C_{n}$ is directly proportional to the geodesic lengths between their images $g\left(x_{i}, x_{j}\right) \in \mathcal{M}$.

Brightwell and co-workers have proven the above statement for the case when the Lorentzian manifold is Minkowski space [5]. Their result uses the fact that, in $d$-dimensional Minkowski space, the volume $V$ of a region of spacetime bounded by the lightcones of 
two causally connected events is $V=m_{d} \tau^{d}$, where $\tau$ is the longest proper time between those events and $m_{d}$ is a constant. In curved spacetimes (with nonconstant curvature) that relationship breaks down in that (a) any proportionality factor between $V$ and $\tau^{d}$ will be a function of spacetime and (b) the dependence of $V$ on $\tau$ may include additional powers of $\tau$. While it is widely believed that the Myrheim length conjecture is true for curved spacetimes, no proof has been achieved.

Underlying the development of the causal set program is a much wider conjecture, termed the Hauptvermutung, which effectively says that in the appropriate limits, a physically interesting causal set will bring forth all of the structure of an approximately unique spacetime manifold $[3,7]$. This conjecture might be stated in the following way:

Let there be a random process that produces a faithfully embeddable causal set $C$.

Then, if $\exists$ maps $g_{1}$ and $g_{2} \ni g_{1}: C \rightarrow \mathcal{M}_{1}$ and $g_{2}: C \rightarrow \mathcal{M}_{2}$, then $\mathcal{M}_{1}$ and $\mathcal{M}_{2}$ are approximately isometric.

One only requires an approximate isometry to allow for random fluctuations where 'approximately isometric' might mean that the manifolds are close in the sense discussed in [11]. This is the main conjecture of causal set kinematics to which the previously mentioned length conjecture is really just a corollary. Some evidence for the validity of the Hauptvermutung has already been established [12] but a full proof has not yet been formulated. However, if the correspondence between a causal set and a manifold is to be (nearly) unique, then not only should the length of the longest paths produce the geodesic length, but the longest paths themselves should produce the geodesic.

In this work, we look for evidence that the longest paths in faithfully embeddable causal sets approach the appropriate geodesic curve as the density of causal set elements increases. This work also constitutes an indirect test of the Myrheim length conjecture in curved spacetimes, because we use this conjecture to identify which paths might produce the geodesic upon embedding. The details of how this is done are given below.

\section{Computational details}

\subsection{Overall approach}

If we consider points $y_{i}$ in a spacetime manifold $\mathcal{M}$, we can define an interval $I_{\mathcal{M}}$ between two causally related points $y_{\mathrm{p}}$ and $y_{\mathrm{f}}$ as the intersection of the future of the pastmost point $y_{\mathrm{p}}$ with the past of futuremost point $y_{\mathrm{f}}$. Let us also define $S_{\mathcal{M}}$ as the geodesic between $y_{\mathrm{p}}$ and $y_{\mathrm{f}}$. Then, our general approach is as follows: (a) we generate many faithfully embeddable causal sets $C=\left\{x_{i}, \prec\right\}$ containing $N$ elements in the form of intervals $I_{C}\left(x_{1}, x_{N}\right)$ by selecting points in $I_{\mathcal{M}}\left(y_{\mathrm{p}}, y_{\mathrm{f}}\right)$ via a Poisson process; (b) in each $I_{C}$, we sample from the set of longest paths $S_{C}=\left\{s_{j}\right\}$ between $x_{1}$ and $x_{N}$ and determine the average goodness-of-fit between the images of the individual $s_{j}$ sampled in $I_{\mathcal{M}}$ and $S_{\mathcal{M}}$; (c) we then repeat steps (a) and (b) for larger causal sets and examine the behaviour of the average goodness-of-fit as $N$ increases.

\subsection{Random sprinklings}

As stated previously, we generate faithfully embeddable causal sets by selecting points from a manifold via a Poisson process - this is also called a random sprinkling into the manifold. Justification for why this should be a Poisson process is given elsewhere [6-8]. The procedure by which we perform these sprinklings is described in [8], but we briefly explain it here for completeness. 
Table 1. Average number of longest paths $s_{j}$, with standard deviations, versus interval size for 100 trial sprinklings. The notation $a\left(10^{b}\right)$ means $a \times 10^{b}$.

\begin{tabular}{rcc}
\hline$N$ & $\left\langle s_{j}\right\rangle$ & $\sigma_{\text {sd }}$ \\
\hline 100 & $1.00\left(10^{2}\right)$ & $1.80\left(10^{2}\right)$ \\
500 & $2.45\left(10^{4}\right)$ & $1.11\left(10^{5}\right)$ \\
1000 & $9.40\left(10^{5}\right)$ & $3.17\left(10^{6}\right)$ \\
1500 & $2.67\left(10^{8}\right)$ & $1.88\left(10^{9}\right)$ \\
2000 & $2.79\left(10^{8}\right)$ & $9.67\left(10^{8}\right)$ \\
\hline
\end{tabular}

The spacetimes used in this study are given by the metric

$$
\mathrm{d} s^{2}=\Omega^{2} \eta_{\alpha \beta} \mathrm{d} x^{\alpha} \mathrm{d} x^{\beta},
$$

where $\Omega^{2}$ is the conformal factor and $\eta_{\alpha \beta}$ is the Minkowski tensor. The sprinklings are performed by a (double) rejection method similar to the methods described in [6] and [9]. The interval is enclosed in a box and points are randomly selected within this box; if a selected point is outside the interval it is rejected, otherwise it is kept-this is the first rejection. In flat spacetime $\left(\Omega^{2}=1\right)$, this first rejection provides the desired uniform distribution of points. In curved spacetimes, however, remaining points face a second rejection to ensure that the final points are distributed uniformly with respect to the volume form $\Omega^{d}$, where $d$ is the dimension of the spacetime. Each remaining point is associated with a random number $w$ within the range $0<w<\Omega_{\max }^{d}$, where $\Omega_{\max }^{d}$ is the maximum value of the volume from within $I_{\mathcal{M}}$. If $w$ is greater than the value of the volume form evaluated at the point in question, the point is rejected, otherwise, it is kept. This process continues until $N$ points are sprinkled into $I_{\mathcal{M}}$.

When partially ordered according to their causal relations in $I_{\mathcal{M}}$, these $N$ points, without the manifold, comprise a faithfully embeddable causal set interval $I_{C}$. At the end of the sprinkling process, the selected points are sorted according to increasing value of the time coordinate. The corresponding elements in $I_{C}$ are labelled with a number from 1 to $N$. However, keep in mind that, exclusive of $x_{1}$ and $x_{N}$, this natural labelling does not necessarily correspond to an ordering of the elements. For example, element $x_{25}$ may precede element $x_{50}\left(x_{25} \prec x_{50}\right)$ or they may not be related in any way.

\subsection{Finding the paths}

Having formed the causal set interval, the longest paths between $x_{1}$ and $x_{N}$ must be found. Starting with element $x_{1}$ we form the set of elements $D_{1}$ linked to its 'future', that is, $D_{1}=\left\{x_{j} \in I_{C} \mid x_{1} \preceq x_{j}\right\}$. For each of these daughters of $x_{1}$ we calculate the length $L\left(x_{j}\right)$ of the longest path from $x_{j}$ to $x_{N}$ using an algorithm due to Sorkin [10]. We save only those elements of $D_{1}$ for which $L\left(x_{j}\right)$ is maximum. This forms the subset $Y_{D 1}$ of the youngest daughters of $x_{1}$. Every element in $Y_{D 1}$ is in a longest path from $x_{1}$ to $x_{N}$. Then, to complete a longest path we can choose any element of $Y_{D 1}$ and repeat the process until we reach an element for which the only daughter is $x_{N}$.

In general, there are many paths that traverse an interval. In fact, the number of paths can become quite large. Table 1 lists the average number of longest paths (for 100 trials) for various interval sizes $N$ for causal sets generated by sprinkling into (1+1)-dimensional Minkowski space. As the table shows, the number of longest paths is on the order of $10^{8}$ for intervals as small as $N=2000$. With so many paths in each $I_{C}$, it is computationally impractical to compare the image of every longest path from all the sprinklings to $S_{\mathcal{M}}$. 

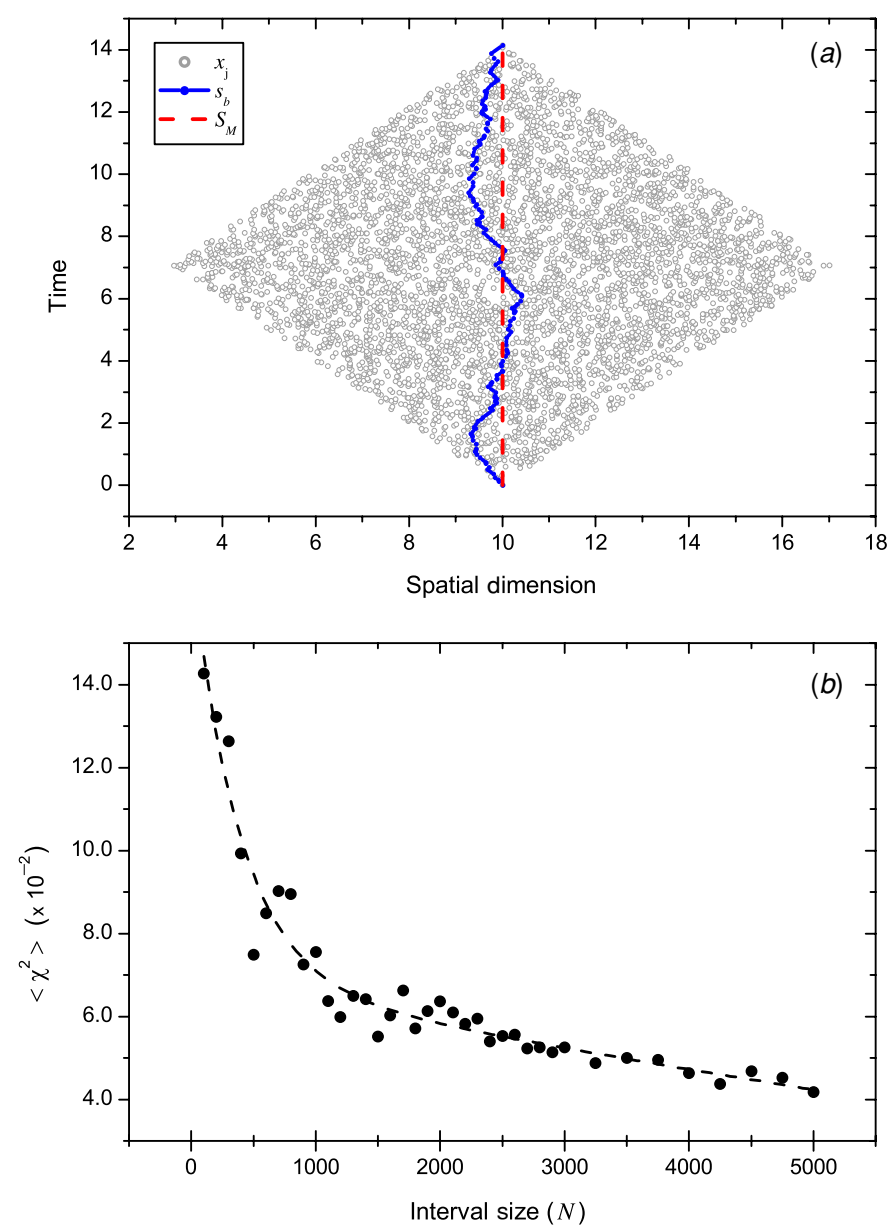

Figure 1. Results for (1+1)-dimensional Minkowski space. Panel $(a)$ shows the sprinkled points $x_{j}$, the geodesic $S_{\mathcal{M}}$ and one of the best longest paths $s_{b}$, determined by the Monte Carlo process described in the text. Panel $(b)$ shows the decrease in $\left\langle\chi^{2}\right\rangle$ versus interval size $N$. The dashed line is just a numerical fit to the data points the purpose of which is to emphasize the trend of the data.

To circumvent this problem, we conduct our study using a Monte Carlo style sampling of the paths in each trial $I_{C}$.

Our procedure is the following. (a) We obtain a trial path $s_{1}$ by selecting the first elements (smallest label) in the various $Y_{D j}$. (b) Using the coordinates from which the elements of $s_{1}$ were sprinkled, we calculate the goodness-of-fit of the image of $s_{1}$ to $S_{\mathcal{M}}$ using a $\chi^{2}$ statistic:

$$
\chi^{2}=\frac{1}{n(d-1)} \sum_{j=1}^{n} \sum_{k=1}^{d-1} \frac{\left(x_{j}^{k}-y_{j}^{k}\right)^{2}}{y_{j}^{k}},
$$

where $n$ is the number of elements in the longest path, $d-1$ is the number of spatial dimensions of the manifold, $x_{j}^{k}$ are the spatial coordinates of points in the image of the path $s_{1}$ in $\mathcal{M}$ and $y_{j}^{k}$ are the spatial coordinates of those points along the geodesic having the same time coordinate as the point with $x_{j}^{k}$. So, in standard chi-squared language, the points from the path play the role of the 'observed' values, the points from the geodesic play the role of the 'expected' 

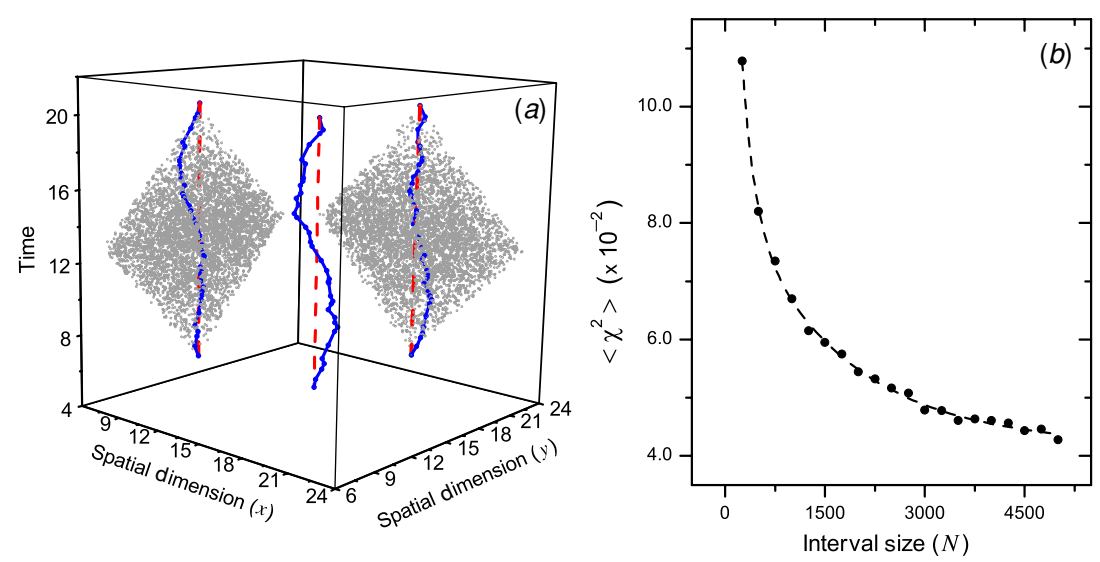

Figure 2. Results for (2+1)-dimensional Minkowski space. Panel (a) shows $x$ - $t$ and $y$ - $t$ projections of the sprinkled points, the geodesic and its projections, and one of the best longest paths and its projections. Panel $(b)$ shows the decrease in $\left\langle\chi^{2}\right\rangle$ versus interval size $N$. The dashed line emphasizes the trend of the data.

values and $n$ is the number of 'degrees of freedom'. (c) We then find a new path by randomly choosing any element, from among all the elements that are along any longest path, that has more than one youngest daughter, randomly choosing one of the youngest daughters, and then completing the path with the first elements of the remaining $Y_{D j}$. (d) We calculate and store $\chi^{2}$ for this new path. The elements of this path are also stored if it is a better fit than the previous path, otherwise they are discarded. (e) We repeat steps (c) and (d) for a number of trials equal to $5 \times L\left(x_{1}\right)$. The prescription for the number of trials is adopted so that this number automatically scales upwards as the number of elements in the causal set, and therefore the expected number of longest paths, increases; the constant 5 is found to be sufficient to produce the best results. The best fit $s_{j}$ that emerges from this process for each trial $I_{C}$ is stored for use in the figures described in the following section. In flat spacetimes small enough that all of the longest paths can be checked, this procedure successfully found the absolute best fit in most of the cases and nearly so in the others [13]. We conduct multiple trial sprinklings (typically 100 for flat spacetimes and 1000 for curved) for a given size $N$ and compute the average of the $\chi^{2}$ values for each $s_{j}$ sampled.

It is worth pointing out that there is a sense in which this approach attempts to mimic the role of an eventual causal set dynamics. One expects that a sum-over-causal sets dynamics should reveal a statistical preference for paths near the geodesic in much the same way as the sum-over-histories approach in quantum mechanics reveals a statistical preference for classical trajectories. To gauge whether the expected longest path does approach the geodesic in the large $N$ limit, we study the behaviour of the average chi-squared $\left\langle\chi^{2}\right\rangle$ as a function of $N$. What we expect is that, if the conjectures hold, the expected longest path will show consistently better fits as $N$ increases. It is important to keep in mind that it is the trend of the fits that matters here more so than the fits themselves.

In flat spacetimes, it is straightforward to identify the actual geodesic curves $S_{\mathcal{M}}$. For curved spacetimes, the $S_{\mathcal{M}}$ are found numerically using the shooting method. The analytical details of how the geodesic equations are set up for doing these calculations are given elsewhere [14]. The specific coordinates $y_{j}^{k}$ used to calculate the $\chi^{2}$ values in equation (2) are then found by interpolating between the numerically calculated points. 

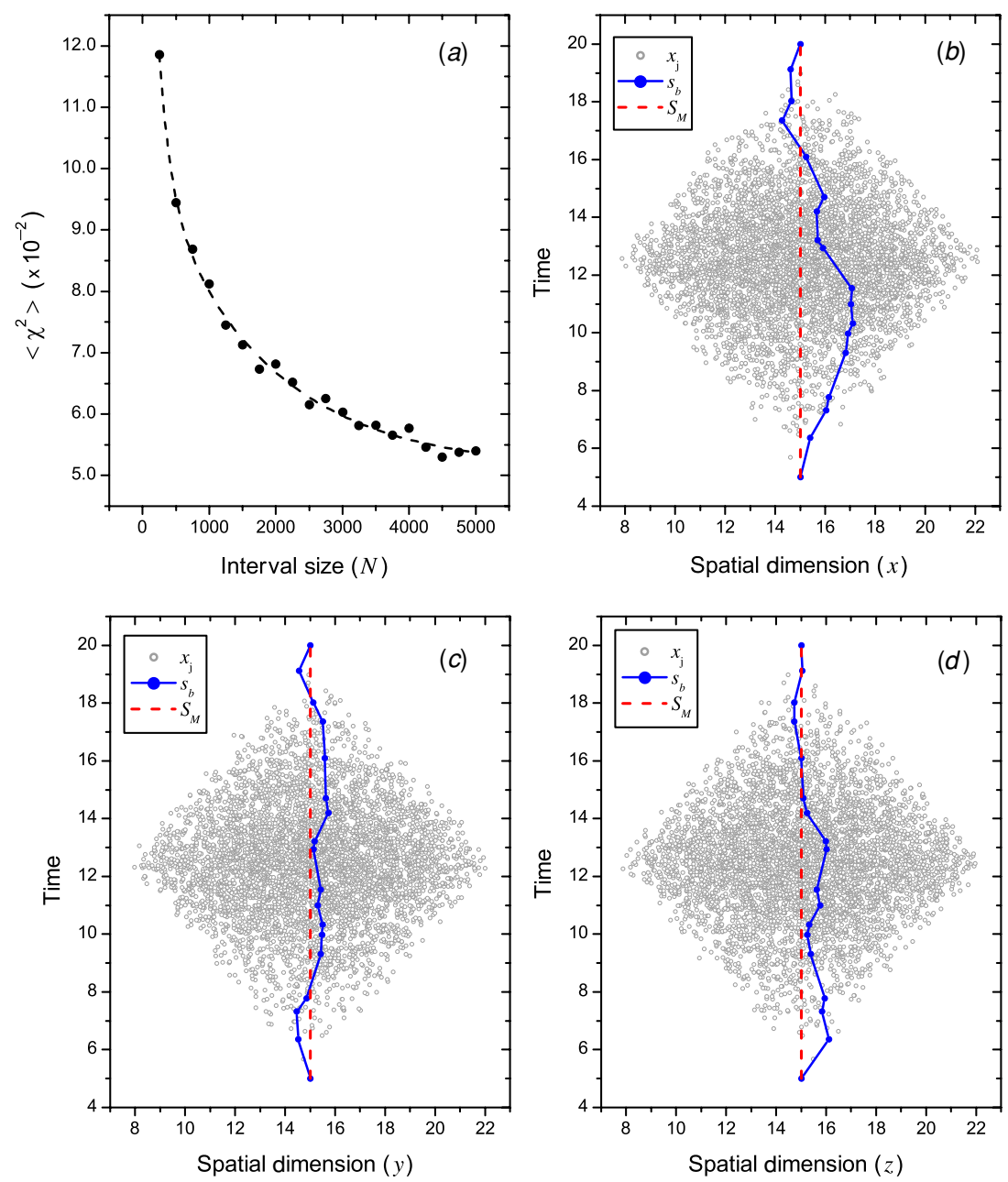

Figure 3. Results for (3+1)-dimensional Minkowski space. Panel (a) shows the decrease in $\left\langle\chi^{2}\right\rangle$ versus interval size $N$ with a dashed line to emphasize the decreasing trend of the data. Panels (b) through $(d)$ show projections of the sprinkled points, the geodesic and one of the best longest paths.

\section{Results and conclusions}

\subsection{Flat spacetimes}

Figure 1 shows the results for (1+1)-dimensional Minkowski space. Panel $(a)$ shows a uniform sprinkling of 5000 points into an interval together with the geodesic $S_{\mathcal{M}}$ and a best longest path $s_{b}$ determined from the Monte Carlo process described previously. We can see from this plot that, modulo statistical fluctuations, $s_{b}$ produces a reasonably good visual fit to $S_{\mathcal{M}}$. However, it is the decreasing trend of $\left\langle\chi^{2}\right\rangle$ in panel $(b)$ of this figure that clearly establishes the key result that the expected longest path in the interval approaches the geodesic as the number of causal set elements increases. Similar results for $(2+1)$ - and (3+1)-dimensional Minkowski spaces are shown in figures 2 and 3, respectively. For those cases (figures $2(a), 3(b)-(d)$ ) only 

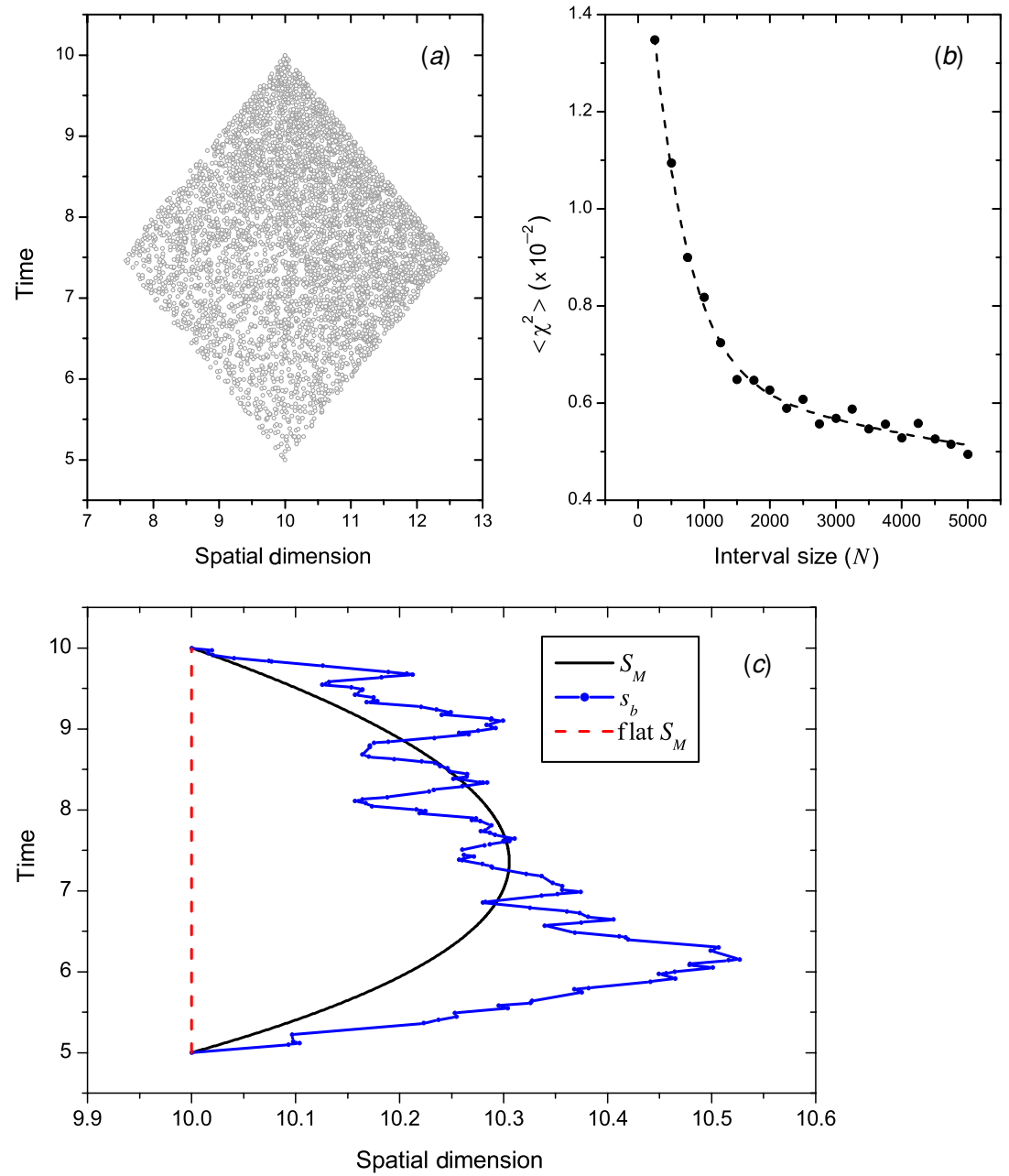

Figure 4. Results for (1+1)-dimensional curved spacetime using the metric of equation (1) with conformal factor $\Omega^{2}=(x t)^{2}$. Panel $(a)$ shows the uniform sprinkling of 5000 points into an interval. Panel $(b)$ shows the decrease in $\left\langle\chi^{2}\right\rangle$ versus interval size $N$. Panel $(c)$ compares one of the best longest paths, $s_{b}$, to the geodesics, $S_{\mathcal{M}}$, for both the curved spacetime and the $(1+1)$ dimensional flat spacetime.

projections of uniform sprinklings are shown. In those projections, the points do not visually appear to be as uniform as in figure 1 $(a)$ because in the higher dimensional cases there is much more volume near the centre of the interval than near $x_{1}$ and $x_{N}$ causing more of the projected points to be centrally located. As with the (1+1)-dimensional case, we can clearly see in figures $2(b)$ and $3(a)$ that $\left\langle\chi^{2}\right\rangle$ decreases as $N$ increases. These flat spacetime calculations constitute direct numerical evidence in favour of the conclusions reached by Brightwell and co-workers [5].

\subsection{Curved spacetimes}

Figure 4 shows the results for a (1+1)-dimensional conformally flat spacetime with conformal factor $\Omega^{2}=(x t)^{2}$. Panel $(a)$ shows a uniform sprinkling of 5000 points. Careful inspection 

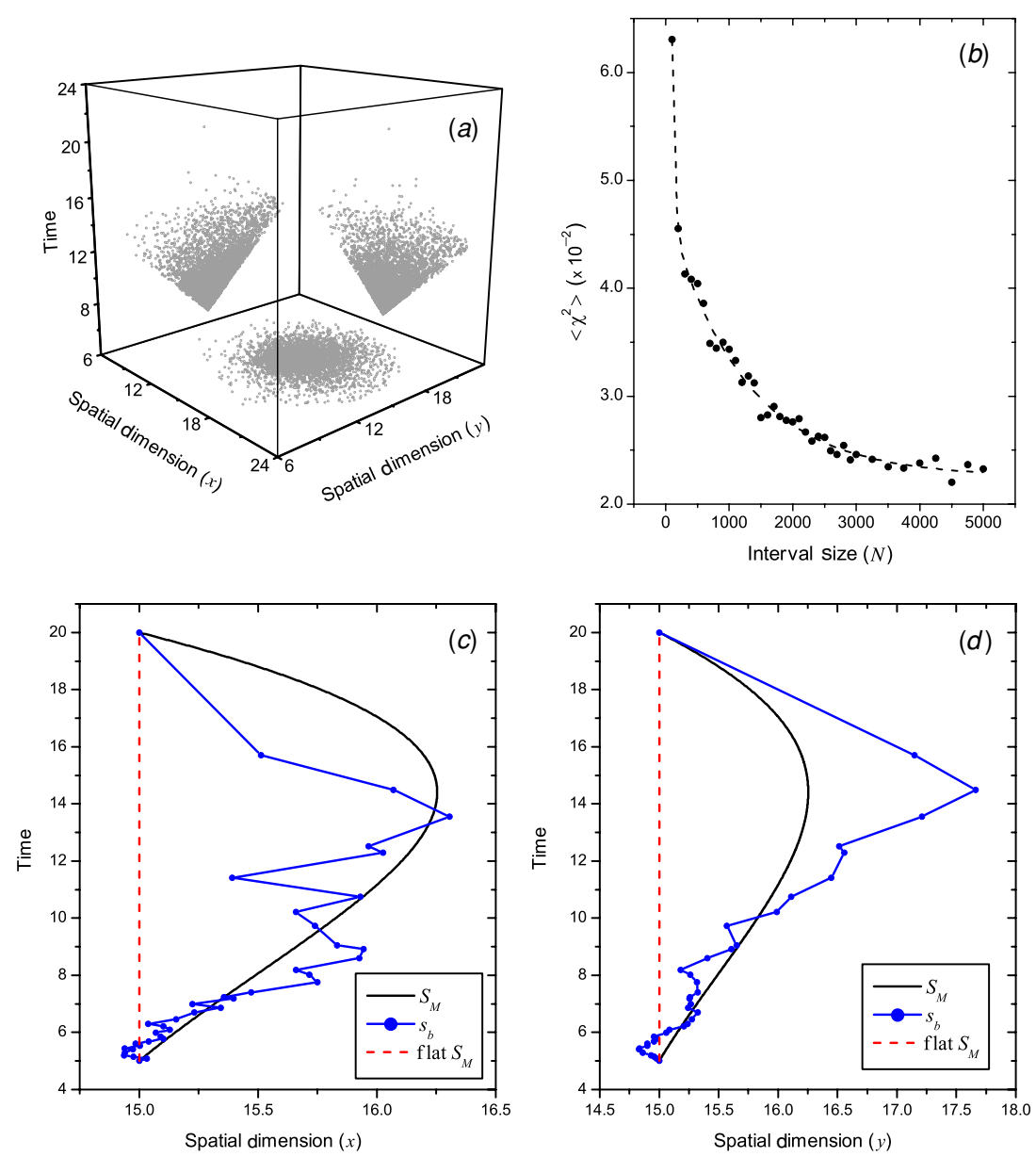

Figure 5. Results for (2+1)-dimensional curved spacetime using the metric of equation (1) with conformal factor $\Omega^{2}=\left(x^{4}+y^{4}\right) / t^{6}$. Panel $(a)$ shows $x-t, y-t$ and $x-y$ projections of a uniform sprinkling of 5000 points. Panel $(b)$ shows the decrease in $\left\langle\chi^{2}\right\rangle$ versus interval size $N$. Panels $(c)$ and $(d)$ compare projections of one of the best longest paths, $s_{b}$, to those of the geodesics, $S_{\mathcal{M}}$, for both the curved spacetime and the (2+1)-dimensional flat spacetime.

of this sprinkling shows that most of the volume (and therefore the points) occur at larger values of $x$ and $t$. Panel $(b)$ shows the key result that the expected longest path approaches the geodesic curve as $N$ increases. For a visual sense of what occurs in this spacetime, panel (c) compares $S_{\mathcal{M}}$ with a best longest path $s_{b}$, also showing the corresponding flat spacetime geodesic for comparison. This figure shows that this $s_{b}$ does appear to follow $S_{\mathcal{M}}$, especially at larger times where there are many more points in the sprinkling.

Figure 5 shows the results for a $(2+1)$-dimensional conformally flat spacetime with conformal factor $\Omega^{2}=\left(x^{4}+y^{4}\right) / t^{6}$. Panel $(a)$ shows the projections of a uniform sprinkling of 5000 points. These projections show that most of the volume occurs at smaller times and larger values of $x$ and $y$. Panel $(b)$ shows the key result that the expected longest path approaches the geodesic curve as $N$ increases. In panels $(c)$ and $(d)$, we see the $x-t$ and $y-t$ projections of $S_{\mathcal{M}}$ and $s_{b}$. In a similar way as in the $1+1$ case, we see that at smaller times, 

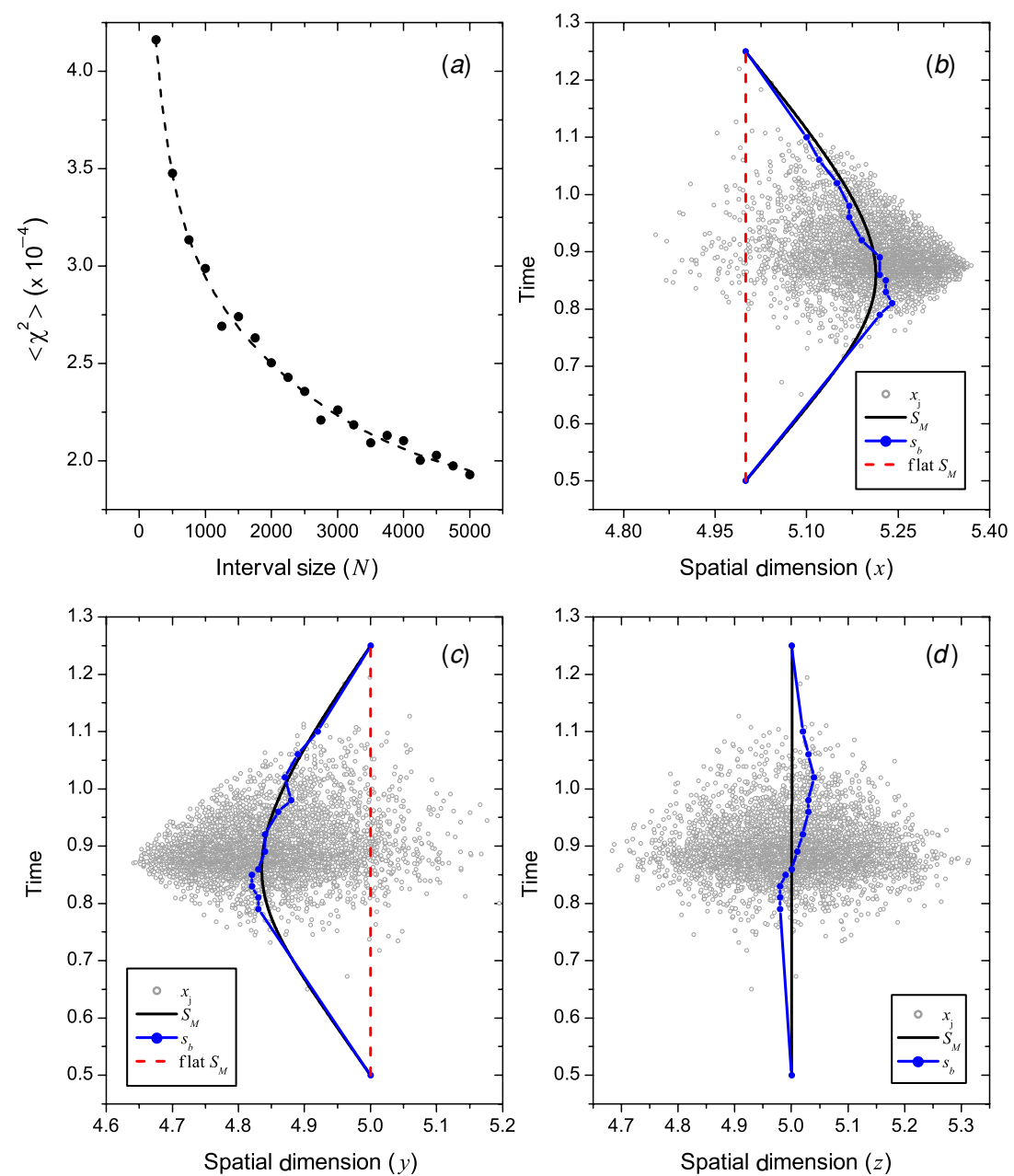

Figure 6. Results for (3+1)-dimensional curved spacetime using the metric of equation (1) with conformal factor $\Omega^{2}=t^{4} \mathrm{e}^{x^{2}-8 y}$. Panel (a) shows the decrease in $\left\langle\chi^{2}\right\rangle$ versus interval size $N$. Panels $(b)$ through $(d)$ show projections of the sprinkled points and compares projections of one of the best longest paths, $s_{b}$, to those of the geodesics, $S_{\mathcal{M}}$, for both the curved spacetime and the (3+1)-dimensional flat spacetime.

where most of the points are, the best longest path does appear to follow along the geodesic curve.

Figure 6 shows the results for a $(3+1)$-dimensional conformally flat spacetime with conformal factor $\Omega^{2}=t^{4} \mathrm{e}^{x^{2}-8 y}$. This conformal factor increases with $x$ and $t$, decreases with $y$ and has no $z$ dependence. Therefore, the features of the interval are that there is more volume at larger values of $x$ and $t$, smaller values of $y$ and uniform in $z$. Projections of a uniform sprinkling of 5000 points into an interval for this spacetime, shown in panels $(b),(c)$ and $(d)$, reveal these features of the interval. Panel $(a)$ confirms that even for this spacetime, the expected longest path approaches the geodesic curve as $N$ increases. This fact is supported visually by panels $(b),(c)$ and $(d)$ which also show that our best longest path is an excellent fit to the geodesic curve. 


\subsection{Conclusions}

In this paper, we have provided computational evidence that the path-geodesic correspondence, implied by key ideas in causal set research, holds for flat and conformally flat curved spacetimes. We further suggest that by establishing that the longest maximal chains approach the actual geodesic curves in the spacetimes we have considered, we have also provided evidence in favour of the wider conjecture, the Hauptvermutung, at the core of causal set kinematics.

\section{Acknowledgments}

We thank Dr Rafael D Sorkin for providing an algorithm for calculating the lengths of paths in a causal set and for further motivation to work on problems of this nature. We are thankful for the helpful suggestions of Natthi L Sharma, Ernest Behringer, James P Sheerin, Daniel W Kittell and Cosmin Ilie. Partial support from the US National Science Foundation is gratefully acknowledged.

\section{References}

[1] Bombelli L, Lee J, Meyer D and Sorkin R D 1987 Phys. Rev. Lett. 59 521-24

Bombelli L, Lee J, Meyer D and Sorkin R D 1988 Phys. Rev. Lett. 60656

[2] Reid D D 2001 Can. J. Phys. 79 1-16

[3] Sorkin R D 2005 Lectures on quantum gravity Proc. Valdivia Summer School (Valdivia, Chile, January 2002) ed A Gomberoff and D Marolf (New York: Plenum)

Dowker F 2005100 Years of Relativity Space-Time Structure: Einstein and Beyond ed A Ashtekar (Hackensack, NJ: World Scientific) (Preprint gr-qc/0508109)

[4] Myrheim J 1978 CERN Preprint TH-2538

[5] Brightwell G and Gregory R 1991 Phys. Rev. Lett. 66 260-3

Bollobas B and Brightwell G 1991 Trans. Am. Math. Soc. 324 59-72

[6] Meyer D 1988 The dimension of causal sets PhD Thesis, MIT

[7] Bombelli L 1987 Space-time as a causal set $P h D$ Thesis, Syracuse University

[8] Reid D D 2003 Phys. Rev. D 67024034

[9] Press W H, Teukolsky S A, Vetterling W T and Flannery B P 1992 Numerical Recipes in Fortran: The Art of Scientific Computing 2nd edn (Cambridge: Cambridge University Press)

[10] Sorkin R D 1998 A library of Lisp functions for posets and other purposes http://physics.syr.edu/ ${ }^{\sim}$ sorkin (version 1.4)

[11] Bombelli L 2000 J. Math. Phys. 41 6944-58

[12] Bombelli L and Meyer D A 1989 Phys. Lett. A 141 226-8

[13] Thompson G B 2003 MS Thesis, Eastern Michigan University

[14] Ilie R 2006 MSc Thesis Eastern Michigan University 